\title{
A SURVEY ON ENERGY EFFICIENT ROUTING PROTOCOLS IN WIRELESS SENSOR NETWORKS
}

By

\section{K.V. PRAVEEN KUMAR *}

M.K. BANGA $* * * *$
B.M. THIPPESWAMY **

V. UDAYA RANI $* * * * *$
S. RESHMA ***

K.R. VENUGOPAL $* * \star * \star *$

* Research Scholar, Department of Computer Science and Engineering, REVA University, Bangalore, India. ** Professor and Head, Department of Computer Science and Engineering, Sambram Institute of Technology. Bangalore, India.

*** Assistant Professor, Department of Computer Science and Engineering, Sambram Institute of Technology. Bangalore, India. $\star \star * \star$ Professor, Department of Computer Science and Engineering, Dayananda Sagar University, Bangalore, India. $\star \star \star \star \star$ Associate Professor, Department of Computer Science and Engineering, REVA University, Bangalore, India. $\star * \star * * *$ Principal, University Visvesvaraya College of Engineering, Bangalore, India.

\section{ABSTRACT}

Energy efficiency is one of the critical issues in the Wireless Sensor Networks (WSNs), since sensor devices are tiny and integrated with a limited capacity battery. In most of the advanced applications, WSNs operate in very harsh areas and not under supervision of human controls. Routing protocols play a significant role in energy balancing by incorporating the techniques that can reduce control overhead, proper data aggregation method and feasible path selection. It demands a unique requirement due to its frequent topology changes and distributive nature. One of the major concerns in the design of routing protocol in WSNs is efficient energy usage and prolonging Network lifetime. This paper mainly discusses different issues related to energy efficiency in routing protocols of all categories. It incorporates most recent routing protocols which improves the energy efficiency in various application environments. This paper also provides comprehensive details of each protocol which emphasize their principles and explore their advantages and limitations. These protocols belong to different classifications based on Network Structures, communication model, topology and Qos parameters. It also includes more relevant and prominent comparisons with all recentState-of-Artworks.

Keywords: Energy Efficiency, Network Lifetime, Routing, Wireless Sensor Networks (WSNs).

\section{INTRODUCTION}

Wireless Sensor Networks (WSNs) consist of a collection of tiny sensing devices, which monitors an event that arises from an area of interest. WSN perhaps operate in a harsh environment with minimum human supervision. Sensor node is a miniature device with little processing power, consisting of sensing unit for data acquisition, little memory for data storage and transceiver for communication with limited power source usually a small battery. WSNs include a wide variety of applications such as, environment monitoring, health monitoring, target tracking, etc. Sensor nodes are deployed for gathering the information and routing it to the sink node or base station. WSNs rely on limited battery power. Saving of power in sensor nodes is a important issue for a prolonged Network lifetime. Design of efficient Routing is the one of the major challenges to minimize the power utilization due to dynamic nature of the WSNs. It is one of the critical issues mainly due to limited transmission range, lack of self-organization and no conventional addressing scheme which makes routing more complex and consume more power. The nodes have a limited and non-replenish battery power due to deployment in harsh areas viz., wild forest, underwater, nuclear fusion, etc. Network lifetime of WSNs mainly depends on the battery capacity of each nodes, optimized dissipation techniques and efficient routing techniques used in a network. Routing algorithms has to carefully manage this scarce resource to increase the lifetime of a sensor network. Even though WSNs are a special category of $\mathrm{AdHoc}$ wireless network, routing techniques of AdHoc networks cannot be used directly due to stringent 
energy constraint requirement in the WSN. Most of the earlier surveys on routing techniques in WSN concentrate on techniques related to maximization of bandwidth, delay constraints and shortest path algorithms. Most of the WSN application scenario requires all the sensor nodes being active for a long time. Hence, energy efficient techniques are most essential in order to avoid the nodes get drained soon. This made the researchers to design energy efficient protocols that minimizes the energy consumption. In this paper, the authors have gathered the most recent information related to energy efficient routing techniques that concentrate on a prolonged network lifetime.

\section{Organization of the Paper}

This paper is organized as follows. Related works is discussed in section 1. Section 2 includes the issues related to energy utilization in WSNs, while section 3 defines the energy efficient route selection parameters. The classifications of routing techniques are presented in section 4. Conclusions are given in the last section.

\section{Related Works}

Several works have been carried out to develop energy efficient routing protocols. Most of the protocols are devised based on different applications and Networks architecture. Some of the most recent works related to this topic are discussed below.

Rahman et al., [1] conducted a comprehensive survey on energy conservation, as it is a most critical issue for WSN. This work concentrates mainly on the design of energy efficient routing protocol that saves and preserves energy of the sensor nodes. It also classifies energy efficient routing protocols and discusses the main advantages and limitations based on limited performance metrics like, bandwidth utilization, QoS parameters and location based information. This survey would have considered some more prominent energy related parameters.

Sheng et al., [2] gathered information on mobile sinks that reduces the hot spot issues in WSNs. Although mobile sinks increase the lifetime of the Networks, it reduces the routing performance of a protocol due to frequent changes in topology and routing paths. This paper classifies the different existing protocols based on the design criteria and discusses the benefits and limitations of these protocols. Still, an exhaustive survey is needed to be considered for low control overhead protocols.

Morteza and Hadi, [3] discussed on energy efficient routing protocols for increasing the lifetime of the Network. They considered well known energy efficient routing algorithms presented based on some important attributes in clustering techniques. The clustering of sensors into group plays an important role in efficient energy consumption in WSNs. The network lifetime can effectively extend by using a precise number of sensor nodes in a cluster environment. This paper discussed only the clustering routing algorithms, but it is also very essential to consider other routing classifications for effective analysis of the energy efficiency for WSNs.

Sudeep et al., [4] presented a detailed survey of hierarchal routing protocols for WSN. It deals with a set of hierarchal protocols, which describes an efficient energy usage for prolonging the lifetime of the Network. This survey gives more useful information about the classification of linear sensor networks. And also it incorporates detailed discussions of linear and non-linear Hierarchal routing protocols in WSNs.

Aslam et al., [5] carried out an extensive survey on energy efficient routing protocols and considered the extension of variations in LEACH based clustering routing protocols. It explains more about the improvement in the network lifetime by using an extended protocol of LEACH. The survey discusses some issues of LEACH with some feasible solutions using variations in LEACH. It also deals with the features and performance issues of hierarchical routing protocols. This paper limits the discussion on energy efficiency, lifetime and data delivery features with analytical comparison only for variations of LEACH.

Theofanis et al., [6] presented the survey on routing protocols for mobile sensor nodes in WSN. It is possible to have few mobile nodes in predominant static WSN to improve network lifetime. It also presents the highlights of benefit and performance issues of the routing techniques with mobile nodes. Discussion of this survey paper has 
been limited to few mobile nodes in static WSNs.

Liu, [7] conducted a comprehensive review of the hierarchal routing protocols. It classifies the hierarchal routing protocols based on different logical topologies. The survey also discussed the benefit and limitation of different hierarchical routing protocols along with significant matrices and application scenarios. It also presents the strategies to select proper topologies for specific applications and provide guidelines to researchers for selecting the appropriate protocols for their application specific network design. Logical topologies plays an important role in WSN to efficiently use the limited resources. This survey neither considers the detailed analytical comparison nor an extensive study of energy efficiency.

\section{Issues Related to Energy Utilization in WSNs}

The WSNs are initially used in military and defense applications and later extended to other advanced applications like surveillance systems in security related issues in banking, battle fields, environmental monitoring and traffic systems [8]. The intrusion detection systems and target tracking problems are also involved in the sensor node applications. [9].

In recent days, the WSNs captured the attention of wide spread applications viz., basic healthcare services [10], smart homes, etc. These applications are used to control and regulate the households like refrigerator, AC, lighting system through Internet of Things (loTs) [1 1]. Sensor nodes are also incorporated in more complex applications related to medical, aerospace engineering and underwater applications [12, 13, 14, 15].

The WSN involves the sensor nodes varying from few nodes to several hundreds based on the type of applications. Each node consists of a control unit to monitor and control with the help of a tiny battery power. It communicates with the help of transceivers through electromagnetic medium with radio frequency ranges. It also associates with different wireless devices to communicate with other sensor nodes in a network. However, each node follows the procedure of respective routing algorithm to transfer the data from the source to the sink. However, an efficient routing algorithm always follows a unique energy utilization model for the overall network performance.

\subsection{Energy Utilization Model for Sensor Nodes in WSNs}

The energy utilization of sensor nodes is measured with respect to the consumption of energy by an individual unit. Each sensor node consists of different units such as, communication unit, sensing unit, power supply unit, and wireless communication unit. Most of the previous works have considered the energy utilization of hardware components along with external radio environment. But these two factors are necessary to view independently.

Wang et al. , [16] proposed a reliable power consumption model for WSNs devices, especially for low power transceivers. It describes that, a single hop routing is more advantageous for typical hardware configurations and radio frequency environments rather than multiple hop routing. The single hop routing greatly minimizes the energy consumption.

Dunkels et al., [1 7] developed a software based on online energy estimation for sensor nodes to perform online accounting in WSNs. It only considers the status of two devices such as, the radio chip and the microcontroller. But it is necessary to consider the whole environment around the sensor node too.

Kellner et al., [18] proposed a more realistic energy model for WSNs, which explain the states and transition of hardware with the help of a set of finite state of machines. It gives more accurate details of the energy consumption in online energy accounting, when compared to earlier works. Here, the online accounting can monitor and refrain the energy consumption of sensor node applications by designing with the help of data gathered by the sensor nodes.

Wang and Yang, [19] discussed about the energy consumption model for power management in WSNs, that depends on the hardware architecture. It considers the details of energy consumption of each hardware unit in a system and implements a communication subsystem energy consumption model. This model indicates the different levels of energy consumption by 
the different hardware devices during the functioning states and transition between the states of devices.

Shareef and Zhu, [20] presented a probabilistic model related to the energy consumption of the processing unit, which is required to assess the level of energy consumption. It appraises the total energy consumption of the processing unit, which includes the energy consumed by all possible states.

\section{Energy Efficient Route Selection Parameters in WSNs}

In WSN, the sensor nodes have a limited scarce resource that has to be used efficiently to extend the lifetime of a sensor network. The existing routing protocols tries using the energy of nodes efficiently by using some parameters such as, stability of link, link distance, transmission power, sleep mode and residual energy. These parameters are used to find an optimal path and to prolong the lifetime of the network. The survivability of WSNs can be improved by considering the novel matrices, while devising the routing protocols.

The main objective of the devising routing protocol is not only finding an optimal path to transmit the data from source to destination, but also to prolong the lifetime of the network. This can be attained by utilizing power-aware routing matrices to improve the performance of the Network.

Even though there is advancement in the hardware technology of the sensor nodes towards optimum energy, we require an energy efficient routing protocol that utilize minimum energy to transmit data from sensor nodes to base station. In WSNs, energy is mainly consumed for sensing, processing and communicating data. These criteria should be considered, while devising routing protocol for WSN. There are some routing matrices that contribute to efficient utilization of the energy and enhance the lifetime of network.

\subsection{Energy Consumption per Packet}

This term is referred to as summation of total power consumed by all intermediate nodes between the source and designation. The energy consumption is proportional to the number of intermediate nodes and distance between the source and the sink.

\subsection{Network Connectivity}

It is defined as the status of the connectivity among each nodes in a network and the ability to communicate with each other. This parameter balances the routing among the cut set to avoid network partition. The network connectivity can be maximized by uniformly balancing the load among all nodes in the network.

\subsection{Variance in Nodes Power Level}

This parameter refers to the difference in power level of each node and expected to maintain minimum difference among all the nodes. The power consumption remains uniform across all the nodes in the network when the load is equally distributed among all the nodes.

\subsection{Node Cost}

Node cost is always inversely proportional to the level of battery charge in a node. The node with less battery power is considered as higher cost and vice versa. Then the cost of each node is considered to devise the routing protocol to improve the network lifetime.

- Network Lifetime:

Network lifetime refers to the duration from the deployment of network to the first node that exhaust its energy completely or all the nodes in the network that exhaust their energies completely.

- Reliability of Network:

It refers to the maximum probability of data delivery to destination within a given time period.

- Average Energy Dissipated:

It refers to the total amount of energy dissipated over a given time period for various functions such as, sensing, transmitting, receiving and data processing in a network.

- EnergyperPacket:

It refers to the total energy required to transmit a packet from the source node to the destination node.

- PacketDeliveryRatio:

It refers to the ratio of total number of packets sent by the source node to the total number of packets received at destination.

- Average PacketDelay: 
It refers to the average latency of a data packet to transmit from the source node to the destination node.

- Idle Listening:

It refers to the state of sensor node in which it is active and consume considerable amount of energy and neither receive nor transmit data packets.

- Back offDelay:

It refers to the time delay incurred for retransmitting the lost data packet.

- NetworkDelay:

It is defined as the total delay incurred for transmission of each data packets from the source to the destination. It includes the delay due to queuing, aggregating and propagation of data packet.

- NetworkThroughput:

It the ratio of the number of packets received by the sink to the number of packets sent from the source in a given unit of time.

- PacketDrop Ratio:

It refers to the ratio of the number of packets sent from the source node to the number of packets received by the destination.

\section{Classification of Routing Techniques in WSNs}

The design of routing techniques is one of the crucial decisive issues in WSNs. The design of routing algorithms requires extensive knowledge of present and future applications to understand its inherent application specific nature. Form the past two decades, several State-of-Art works have proposed various ideas of routing strategies for the betterment of network lifetime, energy efficiency, security and other related QoS parameters. The routing algorithm plays a significant role in WSNs, due to the following reasons.

- Most of the routing designs are application specific and need to fulfill different requirements.

- The method of data transfer is different from traditional networks in such a way that, the data moves from different senor nodes to the common Sink/Base station.

- Optimal recourse management is required to manage the scarce resources like battery power, processor time and memory space.

- An efficient design of routing algorithm is most essential to provide security to different possible attacks like, worm hole attack, sink hole attack and sybil attack.

- The efficient data gathering methods prefer better routing algorithms to maximize data collection based on location information and zone partitioning techniques.

- The routing algorithms should design with an efficient energy and bandwidth utilization strategies to maximize the Network performance.

There are several routing algorithms devised to fulfill the above requirements in the past one and a half decades. But the growing potential in WSNs applications, demand different levels of QoS requirement. However, this paper presents a broad classification of routing algorithms and discuss the most recent State-of-Art works that belongs to this classification. The routing algorithms can be broadly classified into four main categories based on network structure, communication model, topology and reliability as shown in Figure 1.

\subsection{Network Structure}

The distribution of sensor nodes in WSN decides the

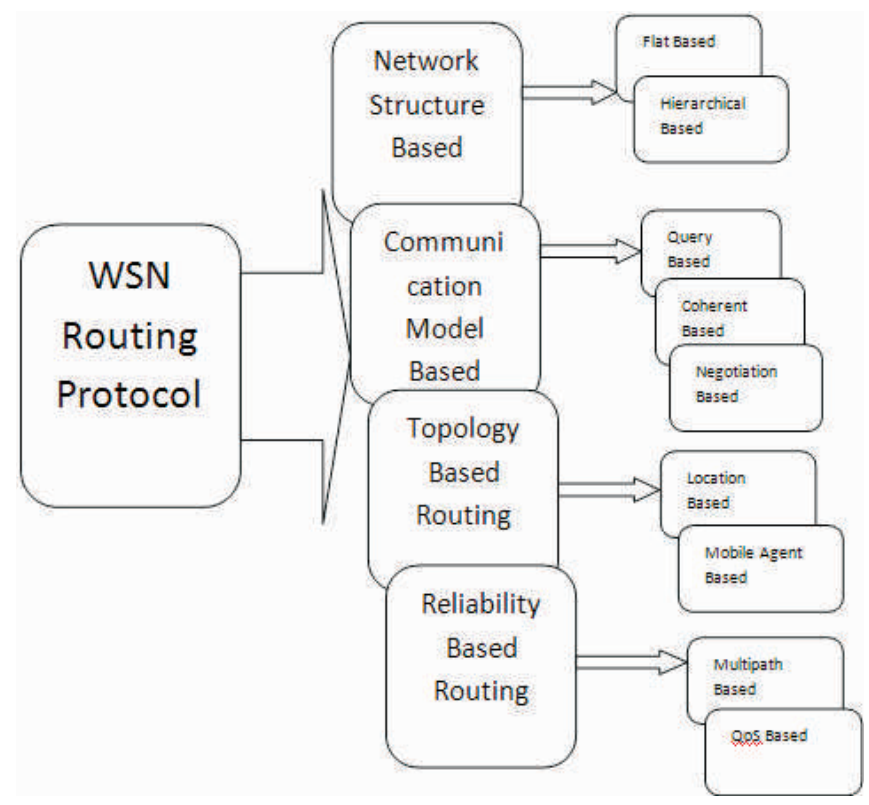

Figure 1. Classification of WSN Routing Protocols 
structure of the network. The structure of the network describes the way in which the nodes are connected with each other. The process of node distribution is based on the type of application and geographical area. Some of the application requires uniform distribution of nodes with equal geographical distance between each node. However, the routing of data in WSN mainly depends on the structure of the network. The network structure is categorized into two sub classes known as, Flat network structure and Hierarchal network structure. The Flat network structure is one in which the nodes are connected at the same level. The Hierarchal network structure organizes all the nodes at different levels.

The routing algorithms related to Flat network structure execute most common responsibilities such as, the resource management and involves minimum time overhead, But these algorithms possess lower network performance compared to hierarchal routing.

The routing algorithms related to hierarchal network structure provides a high energy efficiency and better network performance with greater stability and scalability. These algorithms use clustering techniques to place the nodes at different hierarchal levels. In each clusters, there is a node with higher energy level selected as the cluster head. The cluster head is responsible to collect the data from all its cluster members and forward to the base station through an optimal path. Some of the recent routing protocols related to this category are discussed below.

Vignesh et al., [21] discussed Energy Efficient Routing Protocols using Neighbor Coverage-based Probabilistic Rebroadcast (NCPR) and Good Node Detection Algorithm (GNDA). NCPR is used to find more accurate additional coverage ratio and GNDA selects the energy efficient node that provides the energy efficient network. In order to make an energy efficient network, it is necessary to combine the advantages of the neighbor coverage knowledge and Good node detection algorithm. It reduces the routing overhead delay by decreasing the number of retransmissions. GNDA is used to overcome the energy related problems. The node's energy level is calculated based on the strength of Route
Reply. These two algorithms effectively reduce the routing overhead, number of retransmissions and energy consumption in MANETs. But due to node mobility in MANETs, frequent link breakage leads to higher overhead and reduces the packet delivery ratio, regular path failure and increases the number of packet collisions, especially in dense networks.

Yaakob et al., [22] presented an idea of By-Passing Infected Areas in Wireless Sensor Networks using By-Passed Routing (BPR). This approach avoids the infected areas using fuzzy data clustering method. It mainly solves three main dilemmas in routing i.e., Local minima, visits to unnecessary nodes and false boundary detection. When a node malfunctions, the entire sensing processes may be disturbed and causes serious intermittent connection over the entire network. During such a situation, some packets may not be forwarded to their destinations and become lost in transmission or stuck in the infected areas. Hence, there is a vital need to timely detect the infected nodes and avoid them in subsequent communication and transmission procedures. This requires fast alternative routes to be reconstructed in order to detour any incoming packets to their destinations. But in this approach, the introduction of dynamic routing greatly helps to minimize the possibility of false route diversion that may lead to substantial packet loss and long delays.

Patil et al., [23] proposed an idea of an analysis of various parameters in Wireless sensor networks using Adaptive Forward Error Correction (AFEC) technique. This technique improves the throughput by dynamically tuning FEC with less energy consumption. Adaptive FEC code control algorithm use the higher FEC level after detecting packet loss. Here the transmission overhead for FEC level is less than that of the entire data packet. There are two methods to model bit level propagation errors in packet level simulators, that is, large scale fading and small scale fading. Adaptive FEC code control matches the FEC code size to the low frequency wireless channel BER, which is evaluated by acknowledgement packet arrival. Initially in this algorithm for short distance, the FEC size has minimum number of bytes and for long distance, it is increased to high number of bytes. This paper also 
includes the distance and bandwidth waste ratio analysis and concludes that, it decreases the retransmission traffic, packet loss, delay, error rate and increases the packet delivery ratio by error correction technique. But error correction estimation cost affects the network throughput.

Nidhi and Megha, [24] proposed an idea of practical SMart Grid Networks (SMG). It uses automated modern communication technologies and automated control for effective generation, transmission and distribution of power. These technologies combined both renewable and alternate energy sources that enhance the network lifetime. Smart grid system is implemented using hierarchical architecture to increase the performance of data collection and manage power decapitation. This model consists of several layers and incorporates unique protocols. It utilizes the different communication technologies to transmit information between sensor nodes and electrical appliances. Performance is limited for critical real time applications.

Peng et al., [25] presented the Energy-Efficient Trust System Watchdog Optimizing (ETWO) in WSNs. This technique efficiently schedules the watchdog tasks depending on the nodes' location and trust worthiness of target nodes. Although this technique effectively increases the energy efficiency with forfeit security, it requires an efficient mechanism for reliable data transmission.

Aboobeker et al., [26] devised a Novel Cluster-based Energy Efficient Routing (NCEER) in WSNs. It selects the optimal number of cluster heads to send the data between the source and the sink. The optimality principle is devised in such a way that, the message forwarding consumes minimum energy, when compared to random path. This algorithm exhibit better energy efficiency, packet delivery rate and network lifetime. But it involves more time overhead in transmission of data packets and more energy imbalance in the nodes around the sink node.

Stefanos et al., [27] presented the Energy Efficient Routing protocol for WSNs Through Balancing Cluster (ECHERP).
This technique equalizes the cluster head selection to preserve energy conservation. It amalgamates the method of linear system and Gaussian elimination to choose the cluster heads. Gaussian elimination process the forward elimination technique to find the ranks of the system based on energy spent for operations and back substitution technique to find the solution. It achieves energy efficiency and network lifetime, but QoS are left unnoticed.

Indrajit et al., [28] designed Tree based Energy Efficient Routing (TEER) in WSNs. It uses the L-system cellular automation concept to form the clusters. This algorithm initially arranges all sensor nodes in tree structure and each sub tree form the clusters. Root of the tree act as cluster head which collects data from child node and it transmits the aggregated data to sink node by selecting optimal path using L-system cellular automation scheme. This algorithm efficiently maximizes the lifetime of network. Gaurav Khatana and Manju, [29] addresses Energy Efficient Hierarchical Routing (EEHR) Algorithm for WSNs. This algorithm solves the routing problem for both heterogeneous and homogeneous network based on the attributes such as, reliability and energy-efficiency. It mainly improves the lifetime of the network by prioritizing the sensor nodes according to their remaining power. This algorithm maximizes the network lifetime, but not well suited for time constraint real time applications.

Sohini and Ayan, [30] proposed Energy Efficient Cluster Based Routing Protocol (EECBRP) for heterogeneous WSNs. It considers the remaining energy and distance to sink node for selecting optimal path. This technique balances the load while selecting an the next node to reduce the congestion. It also reduces the link failures and provides solution for infinite loop problem. It is very energy efficient, but throughput reduces as the cluster form is accomplished only after the event is detected.

Abhijith and Sindhu, [31] presented Energy Efficient Multilevel Hierarchical Data Aggregation (EMHD) Mechanism for WSNs. It is a multilevel data aggregation technique, which performs the data aggregation in three different levels of hierarchy. Sensor nodes are categorized 
into low medium and high powered nodes based on energy level and communication range. This technique uses the aggregation at grid level and cluster level to reduce the redundant data transmission which improves the network lifetime.

Zaman et al., [32] presented a Energy Efficient Hierarchal Position Responsive Routing Protocol (PRRP) for WSNs. It divides the network into four quadrants to form the clusters and the cluster head is selected based on the node's energy level. The cluster head employs receiving, transmission and sleep mode to use energy efficiently. It efficiently increases the network throughput by fair distribution data packet among nodes in the network.

Verma and Anuj, [33] presented Hierarchical-Energy Efficient Reactive Network Layer Protocol (H-EERNLP) for WSNs. This algorithm shift the route finding burden from sensor nodes to base station as it comprise abundant resources. It is a most efficient algorithm for dense network, data routing to base station which consider various factors such as code-updater, network monitoring and dynamic programming. The approach is energy efficient but due to traffic load, the gateway node's lifetime is a grave factor.

Zohre, [34] proposed a Hybrid Energy Efficient Routing using a Fuzzy Method (HEER-FM) in WSNs. This algorithm selects node with longest lifetime as cluster heads using a Fuzzy method. Data routing makes use of Earliest First-Tree and Source Initiated Dissemination algorithm to transmit information to the sink node. The approach increases energy efficiency and network lifetime, but limited fuzzy variables are used to select cluster-head and switching between routings.

Hai et al., [35] presented Fan-Shaped Clustering (FSC) protocol. It divides a large-scale network into fan-shaped clusters. This algorithm uses different energy saving technique such as, efficient cluster head selection, relay selection and re-clustering. FSC algorithm improves the energy efficiency, network lifetime and reduces the signaling cost, but density of the network affects the network throughput.

Yanwei et al., [36] proposed an Energy-Efficient Wake-Up
Scheduling for Data Collection and Aggregation (EWSD). It focuses on designing energy-efficient protocols for lowdata-rate WSNs. This algorithm use an efficient centralized and distributed scheduling algorithms to remove the unnecessary listening cost, and reduce the energy cost for state switching and clock synchronization. The node wakeup scheduling considerably reduces the energy consumption, but the delay and control overhead increases.

\subsection{Communication Model}

Communication model in routing protocols dictates the data flow from the source node to the sink node. The protocols belonging to this class, exhibits high delivery ratio with minimum energy consumption. These two parameters are more nearer to theoretical minimum in broadcast and point to point networks. The protocols belongs to this category can be further classified into two sub classes viz., Query based protocols, Coherent and Non coherent based protocols and Negotiation based protocols.

In Query based protocols, the sink requests the data to other nodes in a network by sending a query for the required data. In turn, the node which recognizes this query will send the data to the sink. The coherent and non coherent routing protocols mainly deals with the data aggregation. The coherent protocols perform minimum processing of data before forwarding to the next node, whereas non coherent protocols perform complete preprocessing of the data before forwarding to the aggregation process. Negotiation based protocols are used to minimize the redundancy in data transmission using meta-data negotiations.

Ebenezar and Jayanthy, [37] proposed the improvement of AdHoc On demand Distance Vector (AODV) protocol by Good Node Detection Algorithm (GNDA) for AODV to enhance energy efficiency. GNDA identified the good node for data transfer and stored the good nodes information in the routing table, which exchange among nodes to find optimal path. It improves the overall performance of the network and the network lifetime within the fixed and dynamic transmission range, but data 
loss rate is significantly more in these systems.

Usman et al., [38] proposed an idea of Practical Data Prediction for Real-World (PDPR) Wireless Sensor Networks. This technique uses the derivative based data arrival prediction and suppression rate for data routing. It used the absolute error and relative error to maximize the network lifetime but the data loss rate has been increased.

Zhang and Dong, [39] presented a Virtual CoordinateBased Bypassing Void Routing (BVR-VCM) for WSNs. BVRVCM protocol includes two phases namely, Void Detecting Phase and Virtual Coordinate Mapping Phase. Void detecting phase collects the edge node information around the routing void and virtual coordinate mapping phase maps the edge node coordinates to a virtual circle. The virtual circle effectively reduces the average length of the routing path and transmission delay. Hence increases the network throughput, but discover packets during the detection of larger voids lead to control overhead.

Hariprabhat et al., [40] proposed Energy-Efficient Homogeneous clustering Protocol (EEHP). This protocol judiciously selects the cluster head based on residual energy and utilization of sensor node by its neighbors. It measures geometry and physical location of obstacles to enhance the routing path. This protocol greatly shrinks average hop count to enhance the energy preservation and network lifetime.

Chinh et al., [41] presented a novel optimization algorithm known as Intelligent Water Drops (IWDs) to construct the optimal data aggregation trees for the WSNs. This algorithm arranges sensor nodes into optimal data aggregation tree and optimally selects the aggregation nodes for energy efficient data transfer. The IWD algorithm provides a better data aggregation tree, which improves energy preservation and network lifetime, but the cost of building an optimal tree requires more control and computational overhead.

\subsection{Topology based Protocols}

The WSNs follow dynamic topology strategies due to its inherent limitations like resource scarcity, node failure and routing failures. Many State-of-Art works have been devised in this direction. In topology based routing protocols, all the nodes maintain topology information to which they are bound. These protocols are further classified into two class viz., Location based and Mobile agent based protocols.

Location based protocols are those in which the data is recognized based on location information of each node. These protocols possess maximum energy efficiency by choosing specific path based on location information rather than broadcasting or other methods. But these protocols restrict the scalability factor and involve time overhead in location identification of nodes in a network.

Mobile agent based protocols are those in which a special node acts as a mobile agent. This node moves from one place of the network to other based on requirement. It facilitates to transfer the data from the sensed area to the destination independently and judiciously based on network conditions. These protocols provide more flexibility by removing stringent conditions that were present in earlier networks due to client server environment.

Jalal et al., [42] presented a framework for Evaluating the Best Achievable Performance by Distributed Lifetime Efficient Routing (EBDLER) Schemes. This protocol was designed to find the optimal sink access cost by using distributed Bellman-Ford algorithm and vector of energy consumption rate to enhance the network lifetime and decrease the energy consumption. But this protocol can further enhance by finding an optimal link cost value in the distributed network.

Songtao et al., [43] Design a Concurrent Data Uploading Framework for Mobile Data Gathering (DaGCM) in WSNs. This protocol considers dynamic wireless capacity and power control to minimize the data gathering cost. It splits the data at the transport layer, routing the data at network layer, and power control and compatibility decisions at physical layer to reduce the data collection latency.

Chen et al., [44] presented Energy Efficient Geographic Routing Algorithms (EEGRA) for WSNs. This algorithm incorporate three criteria such as, distance, interference 
and computational cost for finding optimal path by using geographic routing. The routing process integrate the neighbors' identification of each node, power consumption for data transmission and signal-to-noise ratio to measure link cost. The EEGRA enhance the reliability and reduces the energy consumption, but automatic optimal power level setting need to be considered.

Maleq Khan et al., [45] presented Energy-Efficient Routing Schemes for WSNs. This algorithm builds the sub optimal tree by using nearest neighbor tree (EER-NNT). It effectively solves the connectivity problem in the boundary and nodes in spare region. In this algorithm, boundary nodes are connected first and progress towards the dense region to reduce the dead ends. This protocol maximizes the energy efficiency by reducing message complexity.

Hamed et al., [46] proposed Fast Aggregation Scheduling Tree (FAST) in WSNs. It uses the efficient distributed FAST algorithm with minimum time slot for collision free scheduling. The main goal of FAST algorithm is to schedule the aggregation at parent nodes by minimizing the collisions at receiver nodes. This approach reduces latency and provides better data aggregation, but issues such as, data interference and multiple sink environments need to be considered.

Rodolfo et al., [47] addressed Geographic and Opportunistic Routing for Underwater Sensor Networks (GORUSN). It is a anycast routing protocol that uses the greedy forwarding technique to transfer data packets from sensor nodes to multiple sink nodes at sea surface. Data packets are forwarded by considering the current position of the forwarder node and its neighbors. This protocol significantly improves the network performance by reducing void nodes and data traffic. But it reduces the data delivery rate and maximizes the energy cost.

Zhang and Shen, [48] presented a novel online routing scheme known as Energy Efficient Beaconless Geographic Routing (EBGR) in Wireless Sensor Networks. The EBGR provides the loop-free, fully stateless energy efficient routing without prior knowledge of neighbor nodes. It consumes significantly less energy and also improves the unbalanced energy consumption in the network.

\subsection{Reliable Routing Protocols}

Reliable routing protocols are those which can withstand and are stable against frequent routing failures in WSNS. These protocols are devised with better load balancing mechanisms and fulfill the QoS requirements in terms of energy, bandwidth and latency. But these protocols involve time overhead in maintaining and processing routing related information.

Reliable routing protocols are further divided into two sub classes viz., Multipath based protocols and QoS based protocols. Multipath based protocols follow load balancing strategies with greatly minimized route failures during data transmission, whereas QoS based protocols provide better Wuality of Service in terms of Network throughput, Reliability and Latency along with energy efficiency.

Hamed et al., [49] proposed a Score Based Reliable Routing (SBRR) protocol that constructs the best quality path for data transmission. This algorithm selects the best quality routing based on path score parameter, which is determined by four factors such as, energy level, hop count, links error rate and free buffer size of sensor nodes. SBRR maximizes the network lifetime, reliability and data delivery rate, but increases the control overhead.

Indrajeeth et al., [50] presented an idea of optimal reliable Multi-hop virtual multiple-input single-output (VMISO) routing in wireless networks. This algorithm finds the best optimal routing path by using cardinality set curve, which is derived for a high density network. Cardinality set curves are derived by using parameters such as, reliability, data transmission power, and path loss coefficient factors. This algorithm decreases the total energy cost per packet transmission, that increases the network lifetime, but this mechanism involves more complexity while deriving the cardinality set.

Suganya et al., [51] conducted a survey on Energy Multipath Routing (EMR) Protocols for Reliable Data Transmission in Wireless Sensor Networks. The authors have discussed various multipath routing protocols and 
analyzed reliable data transmission in WSNs. This survey made the extensive comparison on performance issues to improve network lifetime and also deals with advantage and limitations of each techniques.

Sandhya et al., [52] developed a Reliable Ad-hoc Ondemand Distance Vector (RAODV) Routing Protocol. It uses the local monitoring of nodes to avoid malicious nodes attack. RAODV protocol detects and isolates the misbehaving nodes in presence of attack, and it also recovers from the same. This protocol enhances the energy efficient network along with security, but increases the control overhead.

Supriya and Rajesh, [53] presented an idea of detecting good neighbor nodes and finding reliable routing paths based on (DNAODV) protocol. This protocol finds the optimal paths by using good neighbor nodes. Good nodes are identified by using criteria like energy level, and free buffer size. This will improve network lifetime and reliable data transmission, but increases the control overhead.

Marcello et al., [54] proposed an idea of Reliability Requirements for Dynamic Structural Monitoring (RRDSM) in Wireless Sensor Networks. It provides reliable data transmission in dynamic routing environment. The adoption of WSNs for dynamic structural monitoring can potentially reduce the costs and risks of monitoring systems.

Sebastin et al., [55] developed an idea of Reliable Energy Efficient Routing (REER) Algorithms in Wireless AdHoc Networks. It provides a reliable path by using the parameters such as, communication cost, remaining battery level and energy consumed to retransmit the packet. This protocol provides the reliable path and effectively reduces the energy consumption. But additional energy-efficient routing techniques are required to prolong the network lifetime and power management.

Antonio et al., [56] analyze the Reliability of Wireless Sensor Networks (RWSN). This algorithm creates the reliability model on the basis on network topology, information about adopted routing and battery levels. This model provides the reliable data transmission and increases the data transmission rate, but it supports only a limited number of communication protocols.

Keontaek et al., [57] addresses Energy Efficient Gathering of Delay Tolerant Sensing Data (EGDTSD) in WSNs. This heuristic algorithm is designed on the basis of insight analysis of the optimal routing patterns. It requires complete coordination among all sensor nodes at sink location and combines the fragmentation along with multipath routing techniques. This process of fragmentation increases the network lifetime, scalability and enhance the energy preservation, but limited to multiple static sink node environment.

Schurgers and Mani, [58] addresses Energy Efficient Routing by providing Practical Guideline for Uniform resource utilization in (EER-PGU) WSNs. This algorithm uses the energy histogram and introduces a scheme that enacts rapidly to random node failures. It compacts multiple streams of data flowing through a route by Data Combining Entity. This algorithm provides high robustness and reduces the overall energy consumption by balancing the data traffic, but it gives less importance for the Quality of Service parameters.

$\mathrm{Xu}$ et al., [59] presented an Energy Management and Cross Layer Optimization (EMCLO) for Wireless Sensor Network Powered by Heterogeneous Energy Sources. It uses the fully distributed and low complexity cross-layer algorithm designed using heterogeneous renewable energy supplies. This algorithm requires knowledge of the instantaneous state of the system. It reduces energy consumption and increases the tradeoff utility, but reduction of delay and modification to the queuing model are required.

Ju Ren et al., [60] presented Lifetime and Energy Hole Evolution Analysis in Data-Gathering (LEHEDG) WSNs. It designs an analytical model to estimate the entire network lifetime, due to the network deployment and boundary of the energy hole in data gathering. This algorithm uses the Double Cost Function based Routing (DCFR), which involves residual energy of nodes and energy consumption rate. It reduces the energy 


\begin{tabular}{|c|c|c|c|c|c|c|c|}
\hline Protocols & Advantages & Disadvantages & Salability & Mobility & Route Metric & $\begin{array}{c}\text { Periodic } \\
\text { Message Type }\end{array}$ & Robust \\
\hline \multicolumn{8}{|c|}{ Network Structure based Protocols } \\
\hline NCPR & $\begin{array}{l}\text { Reduces overhead, retransmission } \\
\text { and energy consumption }\end{array}$ & $\begin{array}{l}\text { More path break reduces } \\
\text { packet delivery ratio and } \\
\text { increases collision }\end{array}$ & Limited & Limited & $\begin{array}{l}\text { Shortest and } \\
\text { reliable }\end{array}$ & Hello exchange & Good \\
\hline BPR & $\begin{array}{l}\text { Detecting and avoiding infected } \\
\text { area for transmission }\end{array}$ & $\begin{array}{l}\text { Substantial packet loss } \\
\text { and long delay }\end{array}$ & Limited & Fixed & $\begin{array}{l}\text { Best route } \\
\text { avoids infected } \\
\text { area }\end{array}$ & Hello exchange & Good \\
\hline AFEC & $\begin{array}{l}\text { Reduces retransmission traffic, packet } \\
\text { loss. Increase packet delivery ratio. }\end{array}$ & $\begin{array}{l}\text { Network throughput } \\
\text { reduces }\end{array}$ & Good & limited & Best route & None & Good \\
\hline SMG & $\begin{array}{l}\text { Increases network lifetime } \\
\text { and reliable. }\end{array}$ & $\begin{array}{l}\text { Limited capacity and not } \\
\text { preferable for critical system }\end{array}$ & Limited & Limited & Reliable route & Hello exchange & Good \\
\hline ETWO & Increases security & $\begin{array}{l}\text { Not well suited for reliable } \\
\text { data transmission }\end{array}$ & Limited & Limited & Secure path & None & Low \\
\hline NCEER & $\begin{array}{l}\text { Achieves good data packet } \\
\text { delivery and network lifetime }\end{array}$ & Increased control over head & Good & Limited & Best route & Hello exchange & Low \\
\hline ECHERP & Increases network life & $\begin{array}{l}\text { QoS and time constraint } \\
\text { are not considered. }\end{array}$ & Limited & Limited & Best route & None & Good \\
\hline EER & Increases network life & More data loss & Good & Limited & Shortest route & None & Low \\
\hline EEAR & Reliable and energy efficient & Propagation delay is more & Limited & No & Best route & Hello exchange & Low \\
\hline EECBRP & Increases network life & Less network throughput & Limited & No & Optimal route & Hello exchange & Low \\
\hline EMHD & $\begin{array}{l}\text { Reduces redundant data transmission } \\
\text { and Increase network life }\end{array}$ & $\begin{array}{l}\text { Not well suited for reliable } \\
\text { data transmission }\end{array}$ & Limited & No & Shortest route & Hello exchange & Low \\
\hline PRRP & $\begin{array}{l}\text { Increases network throughput } \\
\text { and network life }\end{array}$ & Control overhead is more & Limited & No & Reliable route & Hello exchange & Low \\
\hline H-EERNLP & Prolongs the sensor nodes lifetime. & $\begin{array}{c}\text { More prone to partition } \\
\text { of network }\end{array}$ & Good & Limited & Optimal route & None & Low \\
\hline HEER-FM & Increases network life & Control overhead is more & Limited & No & Optimal route & Hello exchange & Low \\
\hline \multicolumn{8}{|c|}{ Communication Model based protocols } \\
\hline GNDA & Improves network throughput & $\begin{array}{c}\text { More prone to partition } \\
\text { of network }\end{array}$ & Good & No & Greedy route & Table exchange & Low \\
\hline PDPR & Increases network lifetime & Data loss rate is more & Good & Good & Optimal route & None & Good \\
\hline BVRVCM & $\begin{array}{c}\text { Reduces the control overhead } \\
\text { and delay }\end{array}$ & Less reliable & Good & Limited & Shortest route & Hello exchange & Low \\
\hline \multicolumn{8}{|c|}{ Topology based protocols } \\
\hline EBDLER & $\begin{array}{l}\text { Increases network lifetime and } \\
\text { decreases energy consumption }\end{array}$ & Less network throughput & Limited & Limited & Optimal route & Table exchange & Low \\
\hline DaGCM & $\begin{array}{l}\text { Reduces data collection latency } \\
\text { and energy }\end{array}$ & $\begin{array}{l}\text { More prone to partition } \\
\text { of network }\end{array}$ & Limited & Good & Optimal route & None & Low \\
\hline EEGRA & $\begin{array}{l}\text { Increases reliability and } \\
\text { reduce consumption }\end{array}$ & Less network throughput & Limited & Limited & Optimal route & Hello exchange & Low \\
\hline EER-NNT & Improves network throughput & Increase message complexity & Good & Limited & Shortest route & Hello exchange & Low \\
\hline FAST & $\begin{array}{l}\text { Reduces latency and improve } \\
\text { data aggregation }\end{array}$ & Less network throughput & Limited & Limited & Shortest route & None & Low \\
\hline GORUSN & Improves network performance & Less data delivery rate & Limited & Limited & Greedy route & Hello exchange & Low \\
\hline \multicolumn{8}{|c|}{ Reliable protocols } \\
\hline SBRR & Provides reliable network & Increase control overhead & Limited & Limited & $\begin{array}{l}\text { Best quality } \\
\text { routing }\end{array}$ & Hello exchange & Good \\
\hline Vmiso & Increase network lifetime & $\begin{array}{l}\text { More Control overhead } \\
\text { and complex }\end{array}$ & Limited & Limited & Best routing & Hello exchange & Low \\
\hline EMR & Increases reliability and throughput & More complex & Limited & Limited & Optimal path & None & Good \\
\hline RAODV & $\begin{array}{l}\text { Improves network lifetime } \\
\text { and provides security }\end{array}$ & $\begin{array}{l}\text { Network performance is } \\
\text { less in dense network }\end{array}$ & Good & Limited & Shortest path & Table exchange & Good \\
\hline DNAODV & $\begin{array}{c}\text { Provides reliable route and } \\
\text { network lifetime }\end{array}$ & $\begin{array}{l}\text { Increase communication } \\
\text { cost }\end{array}$ & Good & Limited & Optimal path & Hello exchange & Good \\
\hline RWDSM & Reliable data transmission & Increase control overhead & Good & Good & Best routing & Hello exchange & Good \\
\hline PAR & Increases throughput & $\begin{array}{l}\text { Control overhead is more } \\
\text { in dense network }\end{array}$ & Good & Limited & Reliable routing & None & Good \\
\hline RSS & Reliable data transmission & More control overhead & Good & Good & Reliable routing & Hello exchange & Good \\
\hline AFECT & Improves throughput & Wastage of bandwidth & Limited & Limited & Best routing & None & Low \\
\hline REER & Improves network reliability & Reduce the throughput & Good & Limited & Reliable routing & None & Good \\
\hline
\end{tabular}




\section{REVIEW PAPERS}

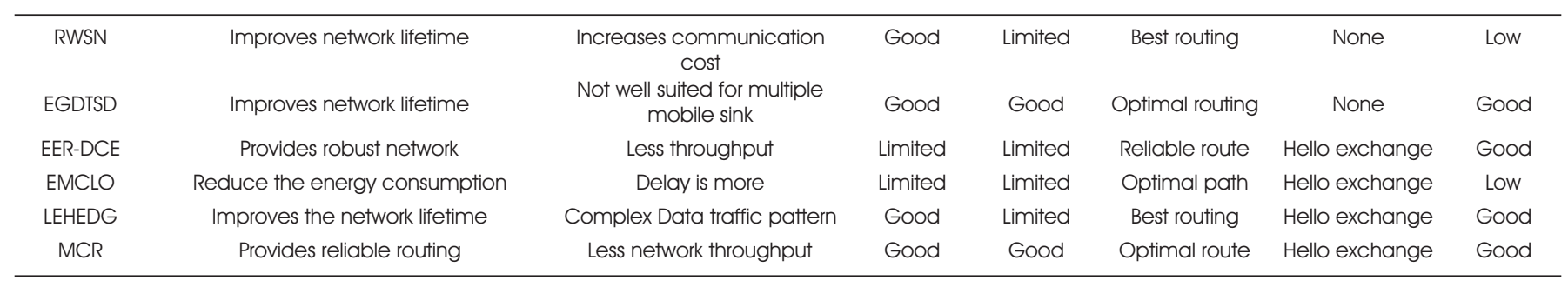

Table 1. Routing Scheme Comparisons

consumption and improves the network lifetime, but lack of analyzing the traffic pattern.

Das et al., [61] addresses Multi Criteria Routing (MCR) for WSN using Weighted Product Model and Relative Rating. It is an efficient multi-hop routing algorithm which selects the best route among the available routes by considering the next hop node using a weighted product model. In this algorithm, weights are calculated based on specific application and relative rating method. The next forwarder node is selected based on residual energy, packet transmission frequency and hop count. This algorithm balances the energy consumption and reduces the delay, but limited data delivery rate.

Tang et al., [62] presented a novel secure and efficient Cost-Aware Secure Routing (CASR) protocol WSNs. This protocol optimizes the network lifetime and security in multi-hop wireless sensor network using impactful parameters such as Energy Balance Control (EBC) and probabilistic based random walking. CASER algorithm is modeled to balance the energy consumption and improve network lifetime along with enhanced security. This algorithm increases the complexity to monitor dropping of energy level and detection of malicious node.

Liu et al., [63] presented the Secret-Sharing-Based Multipath Routing Optimization (SSBMRO). This protocol increases both network security and lifetime using Security and Energy-efficient Disjoint Route (SEDR). It provides major improvement in network security under both single and multiple black hole scenarios without reducing the network lifetime, but it increases the packet loss and delay due to the fading channel.

Table 1 depicts the comparison of the routing schemes which belongs to all the four classifications discussed previously.

\section{Conclusions}

WSNs play an important role in numerous applications and associates with critical issues related to energy efficiency. In this paper, the authors elaborately discussed energy efficient protocols which take major part in solving energy related issues. The discussion pertained to the major classifications of routing algorithms such as, network structure, communication model, topology based and reliability routing. The network structure based protocols solves the issues related to node deployment problem through the sub category viz., flat and hierarchical routing protocols. NCPR, SMG and EEAR are some of the significant protocols which incorporate better routing strategies to improve the energy efficiency compared to other protocols as depicted in Table 1. The communication model based protocols achieves better energy efficiency through protocol strategies such as, negotiation based, coherent and query based techniques. GNDA, PDPR and BVR-VCM are the most recent State-of-Art works designed for higher data delivery rate applications. The next classification is topology based protocols, which include the sub category of mobile agent based and location based protocols. DaDGM, EEGRA and EERNT are better protocols belonging to this category and achieves better energy efficiency for dynamic topology based applications in WSNs. The last category of protocols is reliable routing protocols which provides a better energy efficiency through a sub category namely QoS based protocols and multipath based protocols. SBRR, RAODV, AFECT and LEHEDG are the most recent protocols which play an important role in load balancing environment to maximize the energy efficiency. 


\section{References}

[1]. Md. Atiqur Rahman, Shashed Anwar, Md. lleas Pramanik, and Md. Ferdous Rahman, (2013). "Survey on Energy Efficient Routing Techniques in Wireless Sensor Network". ICACT, pp. 200-205.

[2]. Sheng Yu, Baoxian Zhang, cheng Li, and Hussein T. Mouftah, (2014). "Routing protocols for Wireless Sensor Networks with Mobile Sinks: A Survey". IEEE Communications Magazine, pp. 150-157.

[3]. Morteza M. Zanjireh and Hadi Larijani. A, (2015). "Survey on Centralised and Distributed Clustering Routing Algorithms for WSNs". IEEE, pp. 208-214.

[4]. Sudeep Varshney, Chiranjeev Kumar, and Abhishek Swaroop, (2015). "A Comparative Study of Hierarchical Routing Protocols in Wireless Sensor Networks". IEEE, pp. 1018- 1023.

[5]. M. Aslam, N. Javaid, A. Rahim, U. Nazir, A. Bibi, and Z. A. Khan, (2012). "Survey of Extended LEACH-Based Clustering Routing Protocols for Wireless Sensor Networks". IEEE International Conference on High Performance Computing and Communications, Vol.14, pp.12321239.

[6]. Theofanis P. Lambrou and Christos G. Panayiotou, (2009). "A Survey on Routing Techniques supporting Mobility in Sensor Networks". IEEE International Conference on Mobile Ad-hoc and Sensor Networks, Vol.5, pp. 78-85.

[7]. Xuxun Liu, (2015). "A typical Hierarchical Routing Protocols for Wireless Sensor Networks: A Review". IEEE Sensors Journal.

[8]. E. Shi, and A. Perrig, (2004). "Designing Secure Sensor Networks". IEEE Wireless Commun., Vol.1 1, No.6, pp.3843.

[9]. A. Basharat, N. Catbas, and M. Shah, (2005). "A Framework for Intelligent Sensor Network with Video Camera for Structural Health Monitoring of Bridges". In Proc. $3^{\text {rd }}$ IEEE International Conference on Pervasive Computing and Communications (PerCom), Hawaii, pp.385- 389.

[10]. J. Paek, K. Chintalapudi, R. Govindan, J. Caffrey, and S. Masri, (2005). "A Wireless Sensor Network for Structural Health Monitoring: Performance and Experience". In Proc. $2^{\text {nd }}$ IEEE Workshop on Embedded Networked Sensors EmNetS-II, Syndey Australia, pp. 1-10.

[11]. S. Li, (2006). "Wireless Sensor Actuator Network for Light Monitoring and Control Application". In Proc. IEEE Consumer Communications and Networking Conference, Las Vegas, Vol. 2, pp.974-978.

[12]. C. Lombriser, N. Bharatula, D. Roggen, and G. Troster, (2007). "On-Body Activity Recognition in a Dynamic Sensor Network". In Proc. $2^{\text {nd }}$ International Conference on Body Area Networks (BodyNets), Florence, Italy, Vol. 17, pp. 1-6.

[13]. P. Gibbons, B. Karp, Y. Ke, S. Nath, and S. Seshan, (2003). "IrisNet: An Architecture for a Worldwide Sensor Web". IEEE Pervasive Computing, Vol.2, No.4, pp.22-33.

[14]. M. Srivastava, M. Hansen, J. Burke, A. Parker, S. Reddy, G. Saurabh, M. Allman, V. Paxson, and D. Estrin, (2006). "Wireless Urban Sensing System". CENS Technical Report65, pp. 1-20.

[15]. A. Dunkels, R. Gold, S. Marti, A. Pears, and M. Uddenfeldt, (2005). "Janus: An Architecture for Flexible Access to Sensor Nnetwork". In Proc. $1^{\text {st }}$ ACM Workshop on Dynamic Interconnection of Networks, Cologne, Germany, pp.48-52.

[16]. Q. Wang, M. Hempstead, and W. Yang, (2006). "A Realistic Power Consumption Model for Wireless Sensor Network Devices". In Proc. $3^{\text {rd }}$ Annual IEEE Communications Society on Sensor and Ad HoC Communications and Networks, Reston, Vol. 1, pp.286295.

[17]. A. Dunkels, F. Osterlind, N. Tsiftes, and Z. He, (2007). "Software-Based On Line Energy Estimation for Sensor Nodes". In Proc. $4^{\text {th }}$ workshop on Embedded Networked Sensors, New York, USA, pp. 28-32.

[18]. S. Kellner, M. Pink, D. Meier, and E. Blass, (2008). "Towards a Realistic Energy Model for Wireless Sensor Networks". In Proc. $5^{\text {th }}$ Annual Conference on Wireless on Demand Network Systems and Services, Garmisch, pp.97-100.

[19]. Q. Wang, and W. Yang, (2007). "Energy 
Consumption Model for Power Management in Wireless Sensor Networks". In Proc. $4^{\text {th }}$ Annual IEEE Communications Society Conference on Sensor, Mesh and Ad Hoc Communications and Networks, San Diego, pp. 142-151.

[20]. A. Shareef, and Y. Zhu, (2010). "Energy Modeling of Wireless Sensor Nodes Based on Petri Nets". In Proc. 39 International Conference on Parallel Processing, San Diego, pp. 101-110.

[21]. Vignesh M., Sujay E.K., Tharanya M., Saranya R., and D. Jayachandran, (2014). "An energy efficient routing protocol using NCPR and GNDA algorithms". In International Journal of Computer and Communication Technologies, Vol.2, No.5, ISSN No.: 2278-9723.

[22]. N. Yaakob, I. Khalil, H. Kumarage, M. Atiquizzaman, Z. Tari, (2013). "By-passing infected areas in wireless sensor networks using BPR". In IEEE Transactions on Computers, DOI.10.1109/TC 2014-234J400.

[23]. Netrali Patil, Suraj Patil, Prasanth Mishra and Sachin Chavan, (2014). "AODV based improved method for detecting good neighbor nodes with energy efficiency". In International Journal of Affication or Innovation in Engineering and Management, ISSN: 2319-4847, Vol.3, No.4.

[24]. Nidhi Goel and Megha Agarwal, (2015). "Smart grid networks: A state of art review". Signal Processing and Communication (ICSC), International Conference on, pp. $122-126$.

[25]. Peng Zhou, Siwei Jiang, Athirai Irissappane, Jie Zhang, Jianying Zhou and Joseph Chee Ming Teo, (2015). "Towards energy-efficiency trust system through watch dog optimization for WSNs". In IEEE Transactions on Information Forensics and Security, Vol. 10, No.3, pp.613625.

[26]. Aboobaker Sidhik, Koyamparambil Mammu, Ashwani Sharma, Unai Hernandez-Jayo, and Nekane Sainz, (2013). "A novel cluster-based energy efficient routing in wireless sensor networks". In IEEE $27^{\text {th }}$ International Conference on Advanced Information Networking and Applications, DOI 10. 1109/AINA.2013. 106, pp.41-47.
[27]. Stefanos A. Nikolidakis, Dionisis Kandris, Dimitrios D. Vergados and Christos Douligeri S., (2013). "Energy efficienct routing in wireless sensor networks through balanced clustering". In Algorithms, ISSN: 1999-4893, Vol.6, pp.29-42, DOl: 10.3390/A6010029.

[28]. Indrajit Banerjee, Prasenjit Chanak, Biplab Kumar Sikdar and Hafizur Rahaman, (2011). "EER: Energy efficient routing in wireless sensor networks". In Proceedings of the 2011 IEEE Students Technology Symposium, pp.92-97.

[29]. Gaurav Khatana and Manju, (2014). "Energy efficient algorithm for routing problem in wireless sensor networks". In IEEE International Conference on Recent Advances and Innovations in Engineering .

[30]. Sohini Roy and Ayan Kumar Das, (2014). "Energy Efficient Cluster Based Routing Protocol (EECBRP) for wireless sensor network". In First International Conference on Networks and Soft Computing, pp. 26-29.

[31]. Abhijith H.V. and Sindhu M.P., (2015). "Energy efficient multilevel hierarchical data aggregation mechanism for wireless sensor networks". In IEEE International Advance Computing Conference (IACC), pp. 149-153.

[32]. Noor Zaman, Tung Jang Low and Turki Alghamdi, (2014). "Energy efficient routing protocol for wireless sensor network". In Proceedings of ICACT 2014, pp.808814.

[33]. Pushpak Verma and Anuj Kumar Dwivedi, (2015). "HEERNLP: Heirarchical-energy efficient reactive network layer protocol for WSNs". In $2^{\text {nd }}$ International Conference on Computing for Sustainable Global Development, pp. 1709-1711.

[34]. Zohre Arabi, "HERF: A hybrid energy efficient routing using a fuzzy method in wireless sensor networks".

[35]. Hai Lin, Lusheng Wang and Ruoshan Kong, (2015). "Energy efficient clustering protocol for large-scale sensor networks". In IEEE Sensors Journal, Vol. 15, No.12, pp. 7150 7160.

[36]. Yanwei Wu, Xiang-Yang Li, YunHao Liu, Wei Lau, (2010). "Energy efficient wake-up scheduling for data collection and aggregation". In IEEE Transactions on 


\section{REVIEW PAPERS}

Parallel and Distributed Systems, Vol.21, No.2, pp.275287.

[37]. M.R. Ebenezar Jebarani and T. Jayanthy, (2010). "An analysis of various parameters in wireless sensor networks using adaptive FEC techniques". In International Journal of Ad-Hoc, Sensor and Ubiquitous Comouting, Vol.1, No.3.

[38]. Usman Raza, Alessandro Camerra, Army L. Murphy, Themis Palpanas and Gian Pietro Picco, (2015). "Practical data prediction for real-work wireless sensor networks". In IEEE Transactions on Knowledge and Data Engineering, Vol.27, No.8, pp.2231-2244.

[39]. Dejng Zhang and Enqing Dong, (2015). "A virtual coordinate-based bypassing void routing for wireless sensor networks". In IEEE Sensors Journal, Vol.15, No.7, pp.3853-3862.

[40]. Hari Prabhat Gupta, S.V. Rao, Amit Kumar Yadav and Tanima Dutta, (2015). "Geographic routing in clustered wireless sensor networks among obstacles". In IEEE Sensors Journal, Vol. 15, No.5, pp. 2984-2992.

[41]. D. Chinh Hoang, R. Kumar and S Kumar Panda, (2012). "Optimal data aggregation tree in wireless sensor networks based on intelligent water drops algorithm". In IET Wireless Sensor Systems, ISSN: 2043-6386, DOI: 10.1049/IET-WSS. 201 1.0146, Vol.2, No.3, pp. 282-292.

[42]. Jalal Habibi, Amir G. Aghdam and Ali Ghrayeb, (2015). "A framework for evaluating the best achievable performance by distrubted lifetime-efficient routing schemes in wireless sensor networks". In IEEE Transactions on Wireless Communications, Vol.14, No.6, pp.32313246.

[43]. Songtao Guo, Yuanyuan Yang and Cong Wang, (2015). "DaGCM: A concurrent data uploading framework for mobile data gathering in wireless sensor networks". In IEEE Transactions on Mobile Computing, DOI: 10.1109/TMC.2015.2418202.

[44]. Tseng-Yi Chen, Hsin-Wen Wei, Che-Rung Lee, FuNan Huang, Tsan-sheng Hsu and Wei-Kuan Shih, (2012). "EEGRA: Energy efficient geographic routing algorithms for wireless sensor networks". In International Symposium on Pervasive Systems, Algorithms and Networks, DOI:
10.1109/I-SPAN. 2012.22, pp. 104-113.

[45]. Maleq Khan, Gopal Pandurangan and Bharat Bhargava, (2003). "Energy efficient routing schemes for wireless sensor network".

[46]. Hamed Yousefi, Marzieh Malekimajd, Majid Asouri and Ali Movaghar, (2015). "Fast aggregation scheduling in wireless sensor networks". In IEEE Transaction on Wireless Communications, Vol. 14, No.6, pp.3402-3414.

[47]. P Rodolfo W.L., Countinho, Azzegine Boukerche, Luiz F.M. Vieria and Antonio A.F. Loureiro, (2015). "Geographic and opportunistic routing for underwater sensor networks". In IEEE Transactions on Computers, DOl: $10.1109 /$ TC. 2015.2423677 .

[48]. Haibo Zhang and Hong Shen, (2010). "Energyefficient beaconless geographic routing in wireless sensor networks". In IEEE Transactions on Parallel and Distributing Systems, Vol. 21, No.6, pp.881-896.

[49]. Hamed Yousefi, Ali Dabirmoghaddam, Kambiz Mizanian and Amir Hossein Jahangir. "Score based reliable routing in wireless sensor networks".

[50]. Ch Indrajeeth Singh, Krishnachaitanya Katkam and V. Sundar Ratnam, (2012). "Reliable routing in wireless sensor networks". In International Journal of Latest Research in Science and Technology, Vol.1, No.4, pp.408-412.

[51]. S. Suganya, P. Prabaharan and L. Malathi, (2013). In International Journal of Research in Computer and Communication Technology, Vol.2, No.11, pp. 23205156.

[52]. Sandhya Khurana, Neelima Gupta and Nagendra Aneja, (2006). "Reliable ad-hoc on-demand distance vector routing protocol". In Proceedings of the Fifth International Conference on Networking.

[53]. Supriya Bamane and Rajesh Singh, (2014). "Detecting good neighbor nodes and finding reliable routing path based on AODV protocol", In IOSR Journal of Computer Engineering, e-ISSN: 2278-6661, p-ISSN:22788727, Vol. 16, No. 1, pp. 12-19.

[54]. Marcello Cinque, Domenico Cotroneo, Giapaolo De Caro and Massimiliano Palella, (2006). "Reliability 


\section{REVIEW PAPERS}

requirements of wireless sensor networks for dynamic structural monitoring" . International Workshop on Applied Software Reliability (WASR 2006), pp.8-13.

[55]. Sebastin Christhu Raj A, Helensupriya M. and Shanmuga Priya S., (2014). "Reliable energy efficieny routing algorithms in wireless ad-hoc networks". In International Journal of Scientific and Technology Research, ISSN:2277-8616, Vol.3, No.4.

[56]. Antonio Damaso, Nelson Rosa and Paulo Maciel, "Reliability of Wireless Sensor Networks". Open Access Sensor, pp. 15760-85.

[57]. Keontaek Lee, Sunju Park and Seung-Jae Han, (2015). "Energy efficient gathering of delay tolerant sensing data in wireless sensor networks", In Proceedings of ICOIN 2015, pp. 183-188.

[58]. Curt Schurgers and Mani B Srivastava. "Energy efficient routing in wireless sensor networks".

[59]. Weiqiang $\mathrm{Xu}$, Yushu Zhang, Qingjiang Shiand Xiaodong Wang, (2015). "Energy management and cross layer optimization for wireless sensor network powered by heterogeneous energy sources", In IEEE
Transactions on Wireless Communications, Vol. 14, No.5, pp. 2814-2826.

[60]. Ju Ren, Yaoxue Zhang, Kuan Zhang, Anfenf Liu, Jianer Chen and Xuemin, (2014). "Lifetime and energy hole evolution analysis in data gathering wireless sensor networks". In IEEE Transactions on Industrial Informatics, DOI: 10.1109/T11.2015.2411231.

[61]. BBjoy Das, Suman Sankar Bhunia, Sarbani Roy and Nandini Mukherjee, (2015). "Multi-criteria routing in wireless sensor network using weighted protocol model and relative routing". In IEEE Applications and Innovations in Mobile Computing (AIMOC), pp. 132-136.

[62]. Di Tang, Tongtong Li, Jian Ren and Jie Wu, (2005). "Cost-Aware Secure Routing (CASER) protocol design for wireless sensor networks". In IEEE Transactions on Parallel and Distributed Systems, Vol.26, No.4, pp.960-973.

[63]. Anfeng Liu, Zhongming Zheng, Chao Zhang, Zhigang Chen and Xuemin Shen, (2012). "Secure and energy efficient disjoint multipath routing for WSNs". In IEEE Transactions on Vehicular Technology, Vol.61, No.7, pp.3255-3265. 


\section{ABOUT THE AUTHORS}

K.V. Praveen Kumar is an Associate Professor in the Department of Computer Science and Engineering at Sambhram Institute of Technology, Bangalore, India. He received his Bachelor's degree in Computer Science and Engineering from Bangalore University, Bangalore and Master of Technology in Computer Science and Engineering at Visvesvaraya Technological University India. He is presently pursuing his Ph.D programme in the area of Wireless Sensor Networks at REVA University, Bangalore, India. His research interest is in the area of Wireless Sensor Networks.

B.M. Thippeswamy is a Professor and Head of the Department of Computer Science and Engineering at Sambhram Institute of Technology, Bangalore, India. He obtained his B.E in Computer Science and Engineering from Mysore University and M.E degree in Computer Science and Engineering from Bangalore University, Bangalore. He was awarded Ph.D in the area of Wireless Sensor Networks in JNTU Anantpur, India. His research interest is in the area of Wireless Sensor Networks.

S. Reshma is an Assistant Professor in the Department of Computer Science and Engineering at Sambhram Institute of Technology, Bangalore, India. She received her Bachelor's degree in Computer Science and Engineering from Visvesvaraya Technological University and Master of Technology from Visvesvaraya Technological University, Regional Center, Bangalore. She is presently pursuing her Ph.D programme in the area of Wireless Sensor Networks at Visvesvaraya Technological University, Regional Center, Belgaum. Her research interest is in the area of Wireless Sensor Networks.

M.K. Banga is currently the Professor in the Department of Computer Science and Engineering at Dayanandasagar University. He obtained his PhD in Computer Science from Indian Institute of Technology (IITK), Kharagpur. He has a total of around 32 years of experience in Teaching/industries. He has nearly 16 years of teaching experience in various esteemed Engineering colleges and Universities and served at Wipro Technologies for 16 years in various capacities including General Manager and Head of the Architect Academy. He has published many research papers in various National and International Journals. His research interests include Data Networks, Wireless Ad-hoc Network \& Machine Learning and Analytics.

V. Udaya Rani is an Associate Professor in the Department of Computer Science at REVA University Bangalore, India. She obtained her B.E and M.Tech degrees in Computer Science and Engineering, and PhD degree from Mother Teresa University. Her research interests are in the area of Sensor Networks, AdHoc Networks and Image Processing.

K.R. Venugopal is currently the Principal of University Visvesvaraya College of Engineering, Bangalore University, Bangalore, India. He obtained his Bachelor of Engineering from University Visvesvaraya College of Engineering. He received his Masters degree in Computer Science and Automation from Indian Institute of Science Bangalore. He was awarded Ph.D. in Economics from Bangalore University and Ph.D. in Computer Science from Indian Institute of Technology, Madras. He has a distinguished academic career and has degrees in Electronics, Economics, Law, Business Finance, Public Relations, Communications, Industrial Relations, Computer Science and Journalism. He was a Postdoctoral Research Scholar at University of Southern California, USA. He is Fellow of IEEE. He has authored and edited 51 books on Computer Science and Economics, which include Petrodollar and the World Economy, CAptitude, Mastering C, Microprocessor Programming, Mastering C++ and Digital Circuits and Systems, etc, during his three and a half decades of service at University Visveraya College of Engineering. He has over 400 research papers to his credit. His research interests include Computer Networks, Wireless Sensor Networks, Parallel and Distributed Systems, Digital Signal Processing and Data Mining. 